\title{
NEW RESULTS FOR IMPULSIVE FOURTH-ORDER DIFFERENTIAL EQUATIONS
}

\author{
DONGDONG GAO ${ }^{1}$, JIANLI LI ${ }^{2}$, AND ARMIN HADJIAN ${ }^{3 \dagger}$ \\ ${ }^{1}$ Department of Mathematics and Computer Science, Tongling University, \\ Tongling, Anhui 244000, China \\ ${ }^{2}$ Department of Mathematics, Hunan Normal University, Changsha, Hunan \\ 410081, China \\ ${ }^{3}$ Department of Mathematics, Faculty of Basic Sciences, University of Bojnord, \\ P.O. Box 1339, Bojnord 94531, Iran \\ ${ }^{\dagger}$ Corresponding author
}

\begin{abstract}
In this paper, we consider the fourth-order differential equations with impulsive effects. By applying variational methods and critical point theory, some new criteria to guarantee the fourth-order impulsive differential equations have at least two solutions and infinitely many solutions will be given when the impulsive functions require to satisfy the superlinear growth conditions. Moreover, we improve and extend some previous results.
\end{abstract}

AMS (MOS) Subject Classification. 34A15, 34B37, 58E05.

Key Words and Phrases. Impulsive differential equation, Fourth-order, Two solutions, Infinitely many solutions, Variational methods and critical point theory.

\section{INTRODUCTION}

In this paper, we consider the following fourth-order impulsive differential equation:

$$
\left\{\begin{array}{l}
u^{(i v)}(t)+A u^{\prime \prime}(t)+B u(t)=f(t, u(t)), \quad t \neq t_{j}, \text { a.e. } t \in[0, T] \\
\Delta\left(u^{\prime \prime}\left(t_{j}\right)\right)=I_{1 j}\left(u^{\prime}\left(t_{j}\right)\right), \quad j=1,2, \ldots, m \\
-\Delta\left(u^{\prime \prime \prime}\left(t_{j}\right)\right)=I_{2 j}\left(u\left(t_{j}\right)\right), \quad j=1,2, \ldots, m \\
u(0)=u(T)=u^{\prime \prime}\left(0^{+}\right)=u^{\prime \prime}\left(T^{-}\right)=0,
\end{array}\right.
$$

where $A$ and $B$ are real constants, $f:[0, T] \times \mathbb{R} \rightarrow \mathbb{R}$ is a continuous function, the impulsive functions $I_{1 j}, I_{2 j} \in C(\mathbb{R}, \mathbb{R})$ for $1 \leq j \leq m, 0=t_{0}<t_{1}<t_{2}<\cdots<t_{m}<$ $t_{m+1}=T$ and $\Delta\left(u^{\prime \prime}\left(t_{j}\right)\right)=u^{\prime \prime}\left(t_{j}^{+}\right)-u^{\prime \prime}\left(t_{j}^{-}\right), \Delta\left(u^{\prime \prime \prime}\left(t_{j}\right)\right)=u^{\prime \prime \prime}\left(t_{j}^{+}\right)-u^{\prime \prime \prime}\left(t_{j}^{-}\right)$, where the left and right limits of $u^{\prime \prime}\left(t_{j}\right), u^{\prime \prime \prime}\left(t_{j}\right)$ are represented by $u^{\prime \prime}\left(t_{j}^{-}\right), u^{\prime \prime \prime}\left(t_{j}^{-}\right)$and $u^{\prime \prime}\left(t_{j}^{+}\right)$, $u^{\prime \prime \prime}\left(t_{j}^{+}\right)$, at $t=t_{j}, j=1,2, \ldots, m$, respectively. 
Due to a wide range of significant applications in various sciences, such as physics, engineering, chemistry, biology, ecology, control, etc., impulsive fourth-order differential equations, have played the crucial role in above sciences. Their most outstanding advantage is that they can vividly describe the model which suffer sudden changes at certain instants which can not be modeled by the classical differential equations. Owing to this reason, more and more considerations by many people have been paid to study the existence of solutions for impulsive fourth-order differential equations. By means of variational methods and critical point theory, many interesting results on the fourth-order differential equations have presented to our sight, we refer the readers to $[1,2,3,7,8,9,10]$.

More precisely, Sun et al. in [7] have investigated the fourth-order differential equation with impulsive effects as follow:

$$
\left\{\begin{array}{l}
u^{(i v)}(t)+A u^{\prime \prime}(t)+B u(t)=f(t, u(t)), \quad t \neq t_{j}, \text { a.e. } t \in[0, T] \\
\Delta\left(u^{\prime \prime}\left(t_{j}\right)\right)=I_{1 j}\left(u^{\prime}\left(t_{j}\right)\right), \quad j=1,2, \ldots, l, \\
\Delta\left(u^{\prime \prime \prime}\left(t_{j}\right)\right)=I_{2 j}\left(u\left(t_{j}\right)\right), \quad j=1,2, \ldots, l \\
u(0)=u(T)=u^{\prime \prime}\left(0^{+}\right)=u^{\prime \prime}\left(T^{-}\right)=0 .
\end{array}\right.
$$

They have proved that the problem (1.2) has at least one solution and infinitely many solutions by applying variational methods, the main results are as follow:

Theorem 1.1 ([7, Theorem 3.1]). Assume that the following conditions hold:

(H1) There exists a constant $\mu>2$ such that $0<\mu F(t, u) \leq u f(t, u)$ for every $t \in[0, T]$ and $u \in \mathbb{R} \backslash\{0\}$, where $F(t, u)=\int_{0}^{u} f(t, s) d s ;$

(H2) There exist constants $L_{j}>0, j=1,2, \ldots, l$, such that

$$
\left|I_{1 j}(u)-I_{1 j}(v)\right| \leq L_{j}|u-v|, \quad \forall u, v \in \mathbb{R}, j=1,2, \ldots, l,
$$

where $L_{j}$ satisfy $0<\sum_{j=1}^{l} L_{j}<\frac{\mu-2}{2 M_{1}^{2}(\mu+1)}$, and $M_{1}$ is defined in Lemma 2.2 of [7];

(H3) The impulsive functions $I_{2 j}$ satisfy sublinear growth, i.e., there exist constants $a_{2 j}>0, b_{2 j}>0$ and $\gamma_{2 j} \in[0,1), j=1,2, \ldots, l$ such that

$$
\left|I_{2 j}(u)\right| \leq a_{2 j}+b_{2 j}|u|^{\gamma_{2 j}}, \quad \forall u \in \mathbb{R}, j=1,2, \ldots, l ;
$$

(H4) The following inequality

$$
\frac{1}{2 M_{1}^{2}}-\frac{M T^{4}}{\pi^{4} M_{1}^{2} \delta^{2}}-\sum_{j=1}^{l}\left(L_{j}+a_{2 j}+b_{2 j}+\left|I_{1 j}(0)\right|\right)>0
$$

holds, where $M=\sup \{F(t, u): t \in[0, T],|u|=1\}$.

Then, the problem (1.2) has at least one classical solution.

Theorem 1.2 ([7, Theorem 3.2]). Suppose that (H1)-(H4) hold. Moreover, $f(t, u)$ and impulsive functions $I_{1 j}(u), I_{2 j}(u)$ are all odd in $u$. Then, the problem (1.2) has infinitely many classical solutions. 
In [9], Xie and Luo have further studied the following boundary value problem:

$$
\left\{\begin{array}{l}
u^{(i v)}(t)+A u^{\prime \prime}(t)+B u(t)=\lambda f(t, u(t)), \quad t \neq t_{j}, \text { a.e. } t \in[0,1] \\
\Delta\left(u^{\prime \prime}\left(t_{j}\right)\right)=I_{1 j}\left(u^{\prime}\left(t_{j}\right)\right), \quad j=1,2, \ldots, m \\
-\Delta\left(u^{\prime \prime \prime}\left(t_{j}\right)\right)=I_{2 j}\left(u\left(t_{j}\right)\right), \quad j=1,2, \ldots, m \\
u(0)=u(1)=u^{\prime \prime}(0)=u^{\prime \prime}(1)=0
\end{array}\right.
$$

They have proved that the problem (1.3) has at least one solution by applying variational methods, the main result is as follow:

Theorem 1.3 ([9, Theorem 3.1]). Assume that the following condition holds:

(H5) There exist two positive constants $k_{1}$ and $k_{2}$ such that for each $u \in X$

$$
0 \leq \sum_{j=1}^{m} \int_{0}^{u^{\prime}\left(t_{j}\right)} I_{1 j}(s) d s \leq k_{1} \max _{j \in\{1,2, \ldots, m\}}\left|u^{\prime}\left(t_{j}\right)\right|^{2}
$$

and

$$
0 \leq \sum_{j=1}^{m} \int_{0}^{u\left(t_{j}\right)} I_{2 j}(s) d s \leq k_{2} \max _{j \in\{1,2, \ldots, m\}}\left|u\left(t_{j}\right)\right|^{2} .
$$

If there exist constants $c_{1}, c_{2}$ and $c$ satisfying $c_{1}<\sqrt{2 k_{0}} M_{1} c<\sqrt{2 k_{3}} M_{1} c<c_{2}$ and $0<a\left(c_{2}, c\right)<b\left(c_{1}, c\right)$, where $k_{0}=2-\frac{A}{6}+\frac{B}{60}, k_{3}=k_{0}+k_{1}+\frac{1}{4} k_{2}$,

$$
\begin{aligned}
& a\left(c_{2}, c\right)=\frac{\int_{0}^{1} \max _{|u| \leq c_{2}} F(t, u) d t-\int_{0}^{1} F\left(t, u_{1}(t)\right) d t}{c_{2}^{2}-2 k_{3} M_{1}^{2} c^{2}}, \\
& b\left(c_{1}, c\right)=\frac{\int_{0}^{1} F\left(t, u_{1}(t)\right) d t-\int_{0}^{1} \max _{|u| \leq c_{1}} F(t, u) d t}{2 k_{3} M_{1}^{2} c^{2}-c_{1}^{2}},
\end{aligned}
$$

$u_{1}(t)=c t(1-t)$ and $M_{1}=1+\frac{1}{\pi}$, then, for each $\lambda \in\left(\lambda_{1}, \lambda_{2}\right)$, the problem (1.3) has at least one solution $u$ and $\|u\|_{X}<\frac{c_{2}}{M_{1}}$, where $\lambda_{1}=\frac{1}{2 M_{1}^{2} b\left(c_{1}, c\right)}$ and $\lambda_{2}=\frac{1}{2 M_{1}^{2} a\left(c_{2}, c\right)}$.

In [1], Afrouzi et al. also extended problem (1.3), they considered the following boundary value problem:

$$
\left\{\begin{array}{l}
u^{(i v)}(t)+A u^{\prime \prime}(t)+B u(t)=\lambda f(t, u(t))+\mu g(t, u(t)), \quad t \neq t_{j}, \text { a.e. } t \in[0,1] \\
\Delta\left(u^{\prime \prime}\left(t_{j}\right)\right)=I_{1 j}\left(u^{\prime}\left(t_{j}\right)\right), \quad j=1,2, \ldots, m \\
-\Delta\left(u^{\prime \prime \prime}\left(t_{j}\right)\right)=I_{2 j}\left(u\left(t_{j}\right)\right), \quad j=1,2, \ldots, m \\
u(0)=u(1)=u^{\prime \prime}(0)=u^{\prime \prime}(1)=0 .
\end{array}\right.
$$

They have obtained that the problem (1.4) has at least three classical solutions under the above assumption (H5) by using variational methods. 
In [8], Tian and Liu have considered the following Sturm-Liouville boundary value problem:

$$
\left\{\begin{array}{l}
u^{(i v)}(t)-u^{\prime \prime}(t)+u(t)=f(t, u(t)), \quad t \neq t_{j}, \text { a.e. } t \in[0, T], \\
-\Delta\left(u^{\prime \prime \prime}\left(t_{i}\right)\right)=I_{1 i}\left(u\left(t_{i}\right)\right), \quad i=1,2, \ldots, l, \\
\Delta\left(u^{\prime \prime}\left(t_{i}\right)\right)=I_{2 i}\left(u^{\prime}\left(t_{i}\right)\right), \quad i=1,2, \ldots, l, \\
a u(0)-b u^{\prime}(0)=0, \quad c u(T)+d u^{\prime}(T)=0 \\
a u^{\prime \prime}(0)-b u^{\prime \prime \prime}(0)=0, \quad c u^{\prime \prime}(T)+d u^{\prime \prime \prime}(T)=0 .
\end{array}\right.
$$

They have proved that the problem (1.5) has at least one nontrivial solution by applying variational methods, the main result is as follow:

Theorem 1.4 ([8, Theorem 3.1]). Assume that following conditions hold:

(H6) There exist constants $\mu>2$ and $r \geq 0$ such that for $|\xi| \geq r$,

$$
0<\mu F(t, \xi) \leq \xi f(t, \xi)
$$

(H7) The impulsive functions $I_{1 i}$ satisfy sublinear growth, that is, there exist constants $\alpha_{i}>0, \beta_{i}>0$ and $\gamma_{i} \in[0,1), i=1,2, \ldots, l$ such that

$$
\left|I_{1 i}(u)\right| \leq \alpha_{i}+\beta_{i}|u|^{\gamma_{i}}
$$

(H8) The impulsive functions $I_{2 i}, i=1,2, \ldots, l$ are bounded;

(H9) $f(t, u)=o(|u|), I_{1 i}(u)=o(|u|), I_{2 i}(u)=o(|u|)$ as $|u| \rightarrow 0, i=1,2, \ldots, l$.

Then, the problem (1.5) has at least one nontrivial solution.

Motivated by above fact, it is clear to see that the impulsive functions in $[1,7,8,9]$ are required to satisfy the sublinear growth conditions, Lipschitz continuous conditions or bounded conditions. Compared with the corresponding conditions imposed on impulsive functions $I_{1 j}$ and $I_{2 j}$ in the above literatures, in our paper, we suppose that the impulsive functions $I_{1 j}$ and $I_{2 j}$ are required to satisfy the superlinear growth conditions. Under new certain assumptions, we prove that problem (1.1) has at least two solutions and infinitely many solutions by means of variational methods and critical point theory. It is worth pointing out that there is no paper which has considered the existence of solutions for fourth-order differential equations with superlinear impulsive growth conditions up to know, the aim of this paper is to close this gap. Moreover, we also improve and generalize some previous results.

The arrangement of the rest paper is as follows. In Section 2, some preliminaries and results which are applied in the later paper are presented. In Section 3, the main proof of theorems will be vividly shown. In Section 4, corresponding examples are given to illustrate the obtained results in Section 3. 


\section{PRELIMINARIES}

We will assume for the remainder of the paper that $A \leq 0 \leq B$.

Let us recall some basic knowledge first. In the Sobolev space

$$
X:=H^{2}(0, T) \cap H_{0}^{1}(0, T),
$$

we can consider the inner product

$$
(u, v)=\int_{0}^{T} u^{\prime \prime}(t) v^{\prime \prime}(t) d t, \quad \forall u, v \in X,
$$

which induces the norm

$$
\|u\|=\left[\int_{0}^{T}\left|u^{\prime \prime}(t)\right|^{2} d t\right]^{\frac{1}{2}} .
$$

Then we can define the following norm of $X$ as follow

$$
\|u\|_{X}=\left[\int_{0}^{T}\left(\left|u^{\prime \prime}(t)\right|^{2}-A\left|u^{\prime}(t)\right|^{2}+B|u(t)|^{2}\right) d t\right]^{\frac{1}{2}} .
$$

Since $A \leq 0 \leq B$, it is immediate that $\|u\| \leq\|u\|_{X}$.

The usual norm of $C^{1}([0, T])$ and $L^{p}(0, T)$ are defined as follow:

$$
\|u\|_{\infty}=\max \left\{\max _{t \in[0, T]}|u(t)|, \max _{t \in[0, T]}\left|u^{\prime}(t)\right|\right\}, \quad\|u\|_{L^{p}}=\left(\int_{0}^{T} u^{p}(t) d t\right)^{\frac{1}{p}} .
$$

Lemma $2.1([4])$. Let $u \in X$, then $\|u\|_{\infty} \leq M_{1}\|u\|_{X}$, where

$$
M_{1}=\max \left\{\frac{T^{\frac{3}{2}}}{2 \pi}, \sqrt{T}\right\} .
$$

Remark 2.2. It is clear that the $M_{1}$ is more simple in our results than that in $[7,10]$, which is defined as $M_{1}=\max \left\{\frac{T^{\frac{3}{2}}}{\pi \delta}, \frac{\sqrt{T}}{\delta}\left(1+\frac{1}{\pi}\right)\right\}$, where $\delta:=\sqrt{1-\sigma}$ and $\sigma:=\max \left\{\frac{A T^{2}}{\pi^{2}},-\frac{B T^{4}}{\pi^{4}}, \frac{A T^{2}}{\pi^{2}}-\frac{B T^{4}}{\pi^{4}}, 0\right\}$, we know if assume that $A \leq 0 \leq B$, then $\sigma=0$ and $\delta=1$, so $M_{1}=\max \left\{\frac{T^{\frac{3}{2}}}{\pi}, \sqrt{T}\left(1+\frac{1}{\pi}\right)\right\}$.

Remark 2.3. It is also obvious that the $M_{1}$ is better in our results than that in $[1,9]$, which is defined as $M_{1}=1+\frac{1}{\pi}$. However, $M_{1}=1$ in our results if $T=1$. Thus our results generalize and improve some known results.

Remark 2.4. It is also clear that the $M_{1}$ is better in our results than that in [8], which is defined as $M_{1}=\frac{1}{\sqrt{T}}+\sqrt{T}$. When $T=1, M_{1}=2$ in [8], however, $M_{1}=1$ in our results. Thus our results generalize and improve some known results. 
Definition 2.5. A function $u \in X$ is a weak solution of the problem (1.1) if the following equality

$$
\begin{aligned}
\int_{0}^{T}\left(u^{\prime \prime} v^{\prime \prime}-A u^{\prime} v^{\prime}+B u v\right) d t= & -\sum_{j=1}^{m} I_{1 j}\left(u^{\prime}\left(t_{j}\right)\right) v^{\prime}\left(t_{j}\right)-\sum_{j=1}^{m} I_{2 j}\left(u\left(t_{j}\right)\right) v\left(t_{j}\right) \\
& +\int_{0}^{T} f(t, u(t)) v(t) d t
\end{aligned}
$$

holds for every $v \in X$.

Then we consider the functional $\varphi: X \rightarrow \mathbb{R}$ defined by

$$
\varphi(u)=\frac{1}{2}\|u\|_{X}^{2}+\sum_{j=1}^{m} \int_{0}^{u^{\prime}\left(t_{j}\right)} I_{1 j}(t) d t+\sum_{j=1}^{m} \int_{0}^{u\left(t_{j}\right)} I_{2 j}(t) d t-\int_{0}^{T} F(t, u(t)) d t,
$$

where $F(t, u)=\int_{0}^{u} f(t, s) d s$.

Owing to the continuity of $f$ and $I_{i j}, i=1,2$, we immediately deduce that $\varphi$ is continuous and differentiable at any $u \in X$ and

$$
\begin{aligned}
\varphi^{\prime}(u)(v)= & \int_{0}^{T}\left(u^{\prime \prime} v^{\prime \prime}-A u^{\prime} v^{\prime}+B u v\right) d t+\sum_{j=1}^{m} I_{1 j}\left(u^{\prime}\left(t_{j}\right)\right) v^{\prime}\left(t_{j}\right) \\
& +\sum_{j=1}^{m} I_{2 j}\left(u\left(t_{j}\right)\right) v\left(t_{j}\right)-\int_{0}^{T} f(t, u(t)) v(t) d t, \quad \forall v \in X .
\end{aligned}
$$

Hence, a critical point of $\varphi$, defined by (2.1), give us a weak solution of the problem (1.1).

Lemma 2.6. If $u \in X$ is a weak solution of problem (1.1), then $u$ is also a classical solution of (1.1).

Proof. The proof is similar as to the Lemma 2.1 of [7], we omit it here.

Lemma 2.7. Assume that the sequence $\left\{u_{k}\right\}$ converges weakly to $u \in X$, i.e., $u_{k} \rightarrow u$. Then, we have that $\left\{u_{k}\right\}$ converges strongly to $u \in C([0, T], \mathbb{R})$, i.e., $\left\|u_{k}-u\right\|_{\infty} \rightarrow 0$ as $k \rightarrow \infty$.

Definition 2.8. Let $X$ be a Banach space and $\varphi: X \rightarrow \mathbb{R}$. We say $\varphi$ is sequentially weakly lower semi-continuous, if $\liminf _{k \rightarrow+\infty} \varphi\left(u_{k}\right) \geq \varphi(u)$ as $u_{k} \rightarrow u$ in $X$.

Lemma 2.9. The functional $\varphi$ is sequentially weakly lower semi-continuous.

Proof. Since $X$ is a reflexive real Banach space, we may choose a weakly convergent subsequence, we denote $\left\{u_{k}\right\} \in X$ and $u_{k} \rightarrow u$ in $X$. Also, we have $u_{k} \rightarrow u$ uniformly in $C([0, T], \mathbb{R})$ as $k \rightarrow \infty$; that is,

$$
\left\|u_{k}-u\right\|_{\infty} \rightarrow 0, \quad \liminf _{k \rightarrow \infty}\left\|u_{k}\right\|_{X} \geq\|u\|_{X}, \quad \text { as } k \rightarrow \infty
$$


From (2.1), we have

$$
\begin{aligned}
\liminf _{k \rightarrow \infty} \varphi\left(u_{k}\right)= & \liminf _{k \rightarrow \infty}\left[\frac{1}{2}\left\|u_{k}\right\|_{X}^{2}+\sum_{j=1}^{m} \int_{0}^{u_{k}^{\prime}\left(t_{j}\right)} I_{1 j}(t) d t\right. \\
& \left.+\sum_{j=1}^{m} \int_{0}^{u_{k}\left(t_{j}\right)} I_{2 j}(t) d t-\int_{0}^{T} F\left(t, u_{k}(t)\right) d t\right] \\
\geq & \frac{1}{2}\|u\|_{X}^{2}+\sum_{j=1}^{m} \int_{0}^{u^{\prime}\left(t_{j}\right)} I_{1 j}(t) d t \\
& +\sum_{j=1}^{m} \int_{0}^{u\left(t_{j}\right)} I_{2 j}(t) d t-\int_{0}^{T} F(t, u(t)) d t \\
= & \varphi(u),
\end{aligned}
$$

it implies that $\varphi$ is sequentially weakly lower semi-continuous.

Definition 2.10. Let $X$ be a real Banach space. We say $\varphi \in C^{1}(X, \mathbb{R})$ satisfies the Palais-Smale condition (shortly (P.S.)), if every sequence $\left\{u_{j}\right\} \subset X$ for which $\left\{\varphi\left(u_{j}\right)\right\}$ is bounded and $\varphi^{\prime}\left(u_{j}\right) \rightarrow 0$ as $j \rightarrow 0$, possesses a convergent subsequence in $X$.

Our main tools to prove the main results of this paper are the following theorems.

Theorem $2.11([5])$. Let $\varphi \in C^{1}(X, \mathbb{R})$, and $\varphi$ satisfies the (P.S.) condition. Assume that there exist $u_{0}, u_{1} \in X$ and a bounded neighborhood $\Omega$ of $u_{0}$ satisfying $u_{1} \notin \Omega$ and

$$
\inf _{v \in \partial \Omega} \varphi(v)>\max \left\{\varphi\left(u_{0}\right), \varphi\left(u_{1}\right)\right\}
$$

Then, there exists a critical point $u$ of $\varphi$, i.e., $\varphi^{\prime}(u)=0$, with $\varphi(u)>\max \left\{\varphi\left(u_{0}\right), \varphi\left(u_{1}\right)\right\}$.

Theorem 2.12 ([11]). For the functional $\varphi: M \subseteq X \rightarrow \mathbb{R}$ with $M \neq \emptyset, \min _{u \in M} \varphi(u)=$ $\alpha$ has a solution in case the following conditions hold:

(i) $X$ is a real reflexive Banach space;

(ii) $M$ is bounded and weak sequentially closed;

(iii) $\varphi$ is sequentially weakly lower semi-continuous on $M$.

Theorem $2.13([6])$. Let $X$ be an infinite dimensional Banach space, and let $\varphi \in$ $C^{1}(X, \mathbb{R})$ be even, satisfy the (P.S.) condition, and $\varphi(0)=0$. If $X=V \bigoplus \Upsilon$, where $V$ is finite dimensional, and $\varphi$ satisfies:

(i) There exist constants $\rho, \eta>0$ such that $\varphi(u) \geq \eta$ for all $u \in \Upsilon$ with $\|u\|_{X}=\rho$;

(ii) For each finite dimensional subspace $W \subset X$, there is an $R=R(W)$ such that $\varphi \leq 0$ on $W \backslash B_{R}$,

then $\varphi$ possesses an unbounded sequence of critical values. 


\section{MAIN RESULTS}

In this section we present our main results. To be precise, we establish an existence result of at least two solutions, Theorem 3.1, which is based on Theorems 2.11 and 2.12. Finally, we present another existence result of infinitely many solutions, Theorem 3.3, which is based in turn on Theorem 2.13, and we point out a consequence, Corollary 3.4 .

Theorem 3.1. Assume that the following conditions hold:

(A1) There exist $\mu>2, \xi_{i j}>0, i=1,2, j=1,2, \ldots, m$, such that

(i) $I_{i j}(u) u \leq \mu \int_{0}^{u} I_{i j}(s) d s<0$, for $u \in \mathbb{R} \backslash\{0\}$,

(ii) $\int_{0}^{u} I_{i j}(s) d s \geq-\xi_{i j}|u|^{\mu}$, for $u \in \mathbb{R} \backslash\{0\}$;

(A2) There exist positive constants $c_{1}, c_{2}$ and $\delta \in[0,1)$ such that

$$
|f(t, u)| \leq c_{1}+c_{2}|u|^{\delta}
$$

for all $(t, u) \in[0, T] \times \mathbb{R}$;

(A3) $F(t, u) \leq 0$ for all $(t, u) \in[0, T] \times \mathbb{R}$.

Then, the problem (1.1) has at least two solutions.

Proof. It is easy to see that $\varphi \in C^{1}(X, \mathbb{R})$ is an even functional and $\varphi(0)=0$.

Firstly, we need to prove that $\varphi$ satisfies the (P.S.) condition. Let $\left\{u_{k}\right\} \subset X$ such that $\left\{\varphi\left(u_{k}\right)\right\}$ be a bounded sequence and $\lim _{k \rightarrow \infty} \varphi^{\prime}\left(u_{k}\right)=0$. Assume that there exists a constant $Z_{1}>0$ such that

$$
\left|\varphi\left(u_{k}\right)\right| \leq Z_{1}, \quad\left\|\varphi^{\prime}\left(u_{k}\right)\right\|_{X} \leq Z_{1} .
$$

By (2.1) and (A1), we have

$$
\begin{aligned}
\left\|u_{k}\right\|_{X}^{2}= & 2 \varphi\left(u_{k}\right)+2 \int_{0}^{T} F\left(t, u_{k}(t)\right) d t \\
& -2 \sum_{j=1}^{m} \int_{0}^{u_{k}^{\prime}\left(t_{j}\right)} I_{1 j}(t) d t-2 \sum_{j=1}^{m} \int_{0}^{u_{k}\left(t_{j}\right)} I_{2 j}(t) d t \\
\leq & 2 Z_{1}+2 \int_{0}^{T} F\left(t, u_{k}(t)\right) d t \\
& -\frac{2}{\mu} \sum_{j=1}^{m} I_{1 j}\left(u_{k}^{\prime}\left(t_{j}\right)\right) u_{k}^{\prime}\left(t_{j}\right)-\frac{2}{\mu} \sum_{j=1}^{m} I_{2 j}\left(u_{k}\left(t_{j}\right)\right) u_{k}\left(t_{j}\right) .
\end{aligned}
$$

From (2.2), together with the above inequality one has

$$
\begin{aligned}
\left(1-\frac{2}{\mu}\right)\left\|u_{k}\right\|_{X}^{2} \leq & 2 Z_{1}+2 \int_{0}^{T} F\left(t, u_{k}(t)\right) d t \\
& -\frac{2}{\mu} \varphi^{\prime}\left(u_{k}(t)\right)\left(u_{k}(t)\right)-\frac{2}{\mu} \int_{0}^{T} f\left(t, u_{k}(t)\right) u_{k}(t) d t .
\end{aligned}
$$


By (A2), (3.1) and Lemma 2.1, we have

$$
\left(1-\frac{2}{\mu}\right)\left\|u_{k}\right\|_{X}^{2} \leq 2 Z_{1}+\frac{2}{\mu} Z_{1} M_{1}\left\|u_{k}\right\|_{X}+\left(2+\frac{2}{\mu}\right) T\left[c_{1} M_{1}\left\|u_{k}\right\|_{X}+c_{2} M_{1}^{\delta+1}\left\|u_{k}\right\|_{X}^{\delta+1}\right] .
$$

Since $\mu>2$, (3.2) implies that $\left\{u_{k}\right\}$ is bounded in $X$. Since $X$ is a reflexive space, we may choose a weakly convergent subsequence, we denote $\left\{u_{k}\right\}$ and $u_{k} \rightarrow u$ in $X$. Then we will prove that $u_{k} \rightarrow u$ in $X$. In fact, from Lemma 2.7, we have $u_{k} \rightarrow u$ in $C([0, T], \mathbb{R})$ and $u_{k} \rightarrow$ u, a.e. $t \in[0, T]$, so

$$
\left\{\begin{array}{l}
\left(\varphi^{\prime}\left(u_{k}\right)-\varphi^{\prime}(u)\right)\left(u_{k}-u\right) \rightarrow 0, \\
\sum_{j=1}^{m}\left[I_{1 j}\left(u_{k}^{\prime}\left(t_{j}\right)-u^{\prime}\left(t_{j}\right)\right)\right]\left[u_{k}^{\prime}\left(t_{j}\right)-u^{\prime}\left(t_{j}\right)\right] \rightarrow 0, \\
\sum_{j=1}^{m}\left[I_{2 j}\left(u_{k}\left(t_{j}\right)-u\left(t_{j}\right)\right)\right]\left[u_{k}\left(t_{j}\right)-u\left(t_{j}\right)\right] \rightarrow 0, \\
\int_{0}^{T}\left[f\left(t, u_{k}(t)\right)-f(t, u(t))\right]\left[u_{k}(t)-u(t)\right] d t \rightarrow 0, \text { as } k \rightarrow \infty .
\end{array}\right.
$$

Thus, by (2.2) we have

$$
\begin{aligned}
\left\|u_{k}-u\right\|_{X}^{2}= & \left(\varphi^{\prime}\left(u_{k}\right)-\varphi^{\prime}(u)\right)\left(u_{k}-u\right) \\
& -\sum_{j=1}^{m}\left[I_{1 j}\left(u_{k}^{\prime}\left(t_{j}\right)-u^{\prime}\left(t_{j}\right)\right)\right]\left[u_{k}^{\prime}\left(t_{j}\right)-u^{\prime}\left(t_{j}\right)\right] \\
& -\sum_{j=1}^{m}\left[I_{2 j}\left(u_{k}\left(t_{j}\right)-u\left(t_{j}\right)\right)\right]\left[u_{k}\left(t_{j}\right)-u\left(t_{j}\right)\right] \\
& +\int_{0}^{T}\left[f\left(t, u_{k}(t)\right)-f(t, u(t))\right]\left[u_{k}(t)-u(t)\right] d t \rightarrow 0, \quad \text { as } k \rightarrow \infty .
\end{aligned}
$$

We immediately deduce that $\left\|u_{k}-u\right\|_{X} \rightarrow 0$ as $k \rightarrow \infty$, and this implies that $\left\{u_{k}\right\}$ converges strongly to $u \in X$. So $\varphi$ satisfies the (P.S.) condition.

Next, we verify that there exists $M_{2}>0$ such that the functional $\varphi$ has a local minimum $u_{0} \in B_{M_{2}}=\left\{u \in X:\|u\|_{X}<M_{2}\right\}$.

In fact, let $\left\{u_{k}\right\} \subseteq \bar{B}_{M_{2}}$ and $u_{k} \rightarrow u$ as $k \rightarrow \infty$, from Mazur Theorem, there exists a sequence of convex combinations

$$
v_{k}=\sum_{j=1}^{k} \alpha_{k j} u_{j}, \quad \sum_{j=1}^{k} \alpha_{k j}=1, \quad \alpha_{k j} \geq 0, \quad k \in \mathbb{N},
$$

such that $v_{k} \rightarrow u$ in $X$. Since $\bar{B}_{M_{2}}$ is a closed convex set, we have $\left\{v_{k}\right\} \subseteq \bar{B}_{M_{2}}$ and $u \in \bar{B}_{M_{2}}$, thus we proved that $\bar{B}_{M_{2}}$ is bounded and weak sequentially closed.

Since $X$ is a reflexive Banach space and $\varphi$ is sequentially weakly lower semicontinuous on $\bar{B}_{M_{2}}$, by means of Theorem 2.12 , we deduce that $\varphi$ has a local minimum $u_{0} \in \bar{B}_{M_{2}}$. Without loss of generality, we assume that $\varphi\left(u_{0}\right)=\min _{u \in \bar{B}_{M_{2}}} \varphi(u)$. Then we will verify

$$
\varphi\left(u_{0}\right)<\inf _{u \in \partial B_{M_{2}}} \varphi(u)
$$


In fact, let $\varepsilon=M_{2}>0$ satisfying

$$
\frac{1}{2} \varepsilon^{2}-\sum_{j=1}^{m}\left(\xi_{1 j}+\xi_{2 j}\right) M_{1}^{\mu} \varepsilon^{\mu}>0 .
$$

For any $u=\varepsilon \tau$ with $\tau \in X$ and $\|\tau\|_{X}=1$, one has $u \in \partial B_{M_{2}}$. By (2.1), (3.4), (A1) and (A3), we have

$$
\begin{aligned}
\varphi(u)=\varphi(\varepsilon \tau)= & \frac{1}{2}\|\varepsilon \tau\|_{X}^{2}+\sum_{j=1}^{m} \int_{0}^{\varepsilon \tau^{\prime}\left(t_{j}\right)} I_{1 j}(t) d t \\
& +\sum_{j=1}^{m} \int_{0}^{\varepsilon \tau\left(t_{j}\right)} I_{2 j}(t) d t-\int_{0}^{T} F(t, \varepsilon \tau(t)) d t \\
\geq & \frac{1}{2} \varepsilon^{2}-\sum_{j=1}^{m}\left(\xi_{1 j}+\xi_{2 j}\right) M_{1}^{\mu} \varepsilon^{\mu}>0 .
\end{aligned}
$$

Thus, $\varphi(u)>0=\varphi(0) \geq \varphi\left(u_{0}\right)$ for $u \in \partial B_{M_{2}}$, that is, (3.3) holds and $u_{0} \in B_{M_{2}}$.

Finally, we verify that there exists $u_{1}$ with $\left\|u_{1}\right\|>M_{2}$ such that $\varphi\left(u_{1}\right)<$ $\inf _{u \in \partial B_{M_{2}}} \varphi(u)$.

In fact, from (i) of (A1), we deduce that there exist positive constants $d_{1 j}, d_{2 j}$, $D_{1}$ and $D_{2}$ such that

$$
\int_{0}^{u^{\prime}} I_{1 j}(s) d s \leq-d_{1 j}\left|u^{\prime}\right|^{\mu}+D_{1}
$$

and

$$
\int_{0}^{u} I_{2 j}(s) d s \leq-d_{2 j}|u|^{\mu}+D_{2}
$$

for $u \in X$. Now, let $u_{1}=\sigma \theta, \sigma>0$ with $\theta \in X$ and $\|\theta\|_{X}=1$, then by (2.1), (3.5), (3.6) and (A2), we have

$$
\begin{aligned}
\varphi\left(u_{1}\right)=\varphi(\sigma \theta)= & \frac{1}{2}\|\sigma \theta\|_{X}^{2}+\sum_{j=1}^{m} \int_{0}^{\sigma \theta^{\prime}\left(t_{j}\right)} I_{1 j}(t) d t \\
& +\sum_{j=1}^{m} \int_{0}^{\sigma \theta\left(t_{j}\right)} I_{2 j}(t) d t-\int_{0}^{T} F(t, \sigma \theta(t)) d t \\
\leq & \frac{1}{2} \sigma^{2}+T\left[c_{1} M_{1} \sigma+c_{2} M_{1}^{\delta+1} \sigma^{\delta+1}\right] \\
& -\sigma^{\mu}\left[\sum_{j=1}^{m} d_{1 j}\left|\theta^{\prime}(t)\right|^{\mu}+\sum_{j=1}^{m} d_{2 j}|\theta(t)|^{\mu}\right]+m\left(D_{1}+D_{2}\right),
\end{aligned}
$$

then, we deduce that there exists sufficiently large $\sigma>M_{2}=\varepsilon>0$ such that $\varphi(\sigma \theta)<0$. So, we have

$$
\max \left\{\varphi\left(u_{0}\right), \varphi\left(u_{1}\right)\right\}<\inf _{u \in \partial B_{M_{2}}} \varphi(u)
$$


that is, we obtain another critical point $u^{*}$ by Theorem 2.11. Therefore, $u_{0}, u^{*}$ are two critical points of $\varphi$, which are two solutions of (1.1).

Remark 3.2. In our results, the impulsive functions $I_{1 j}$ and $I_{2 j}$ require to satisfy the superlinear growth conditions which are different from $[1,7,8,9,10]$.

Theorem 3.3. Assume that (A1)-(A3) hold and $f(t, u)$ and $I_{i j}(u), i=1,2, j=$ $1,2, \ldots, m$, are odd in $u$. Then, the problem (1.1) has infinitely many solutions.

Proof. Bacause of the continuity of $f$ and $I_{i j}, i=1,2, j=1,2, \ldots, m$, we clearly know that $\varphi$ is continuous and differentiable. Since $f(t, u)$ and $I_{i j}(u), i=1,2$, $j=1,2, \ldots, m$, are odd in $u$, we know $\varphi$ is even and $\varphi(0)=0$. As the similar proof of Theorem 3.1, we obtain that $\varphi$ satisfies the (P.S.) condition. By (2.1), (A1) and (A3), we have

$$
\begin{aligned}
\varphi(u)= & \frac{1}{2}\|u\|_{X}^{2}+\sum_{j=1}^{m} \int_{0}^{u^{\prime}\left(t_{j}\right)} I_{1 j}(t) d t \\
& +\sum_{j=1}^{m} \int_{0}^{u\left(t_{j}\right)} I_{2 j}(t) d t-\int_{0}^{T} F(t, u) d t \\
\geq & \frac{1}{2}\|u\|_{X}^{2}-\sum_{j=1}^{m}\left(\xi_{1 j}+\xi_{2 j}\right) M_{1}^{\mu}\|u\|_{X}^{\mu} .
\end{aligned}
$$

Since $\mu>2$, the above inequality implies that there exists $\rho>0$ small enough such that $\varphi(u) \geq \alpha>0$ with $\|u\|=\rho$. That is, (i) of Theorem 2.13 holds.

For every $\eta>0$ and any finite dimensional subspace $W \subset X$, by (2.1), (3.5), (3.6) and (A2), we have

$$
\begin{aligned}
\varphi(\eta u)= & \frac{1}{2}\|\eta u\|_{X}^{2}+\sum_{j=1}^{m} \int_{0}^{\eta u^{\prime}\left(t_{j}\right)} I_{1 j}(t) d t \\
& +\sum_{j=1}^{m} \int_{0}^{\eta u\left(t_{j}\right)} I_{2 j}(t) d t-\int_{0}^{T} F(t, \eta u(t)) d t \\
\leq & \frac{1}{2} \eta^{2}\|u\|_{X}^{2}+T\left[c_{1} M_{1} \eta\|u\|_{X}+c_{2} M_{1}^{\delta+1} \eta^{\delta+1}\|u\|_{X}^{\delta+1}\right] \\
& -\eta^{\mu}\left[\sum_{j=1}^{m} d_{1 j}\left|u^{\prime}(t)\right|^{\mu}+\sum_{j=1}^{m} d_{2 j}|u(t)|^{\mu}\right]+m\left(D_{1}+D_{2}\right),
\end{aligned}
$$

for $u \in W$. Let $\vartheta(t) \in W$ with $\|\vartheta\|_{X}=1$. From above inequality, we have

$$
\begin{aligned}
\varphi(\eta \vartheta(t)) \leq & \frac{1}{2} \eta^{2}+T\left[c_{1} M_{1} \eta+c_{2} M_{1}^{\delta+1} \eta^{\delta+1}\right] \\
& -\eta^{\mu}\left[\sum_{j=1}^{m} d_{1 j}\left|\vartheta^{\prime}(t)\right|^{\mu}+\sum_{j=1}^{m} d_{2 j}|\vartheta|^{\mu}\right]+m\left(D_{1}+D_{2}\right) .
\end{aligned}
$$


Since $\mu>2$, the above inequality implies that there exists $\eta_{1}>0$ such that $\|\eta \vartheta(t)\|_{X}=$ $\eta>\rho$ and $\varphi(\eta \vartheta(t))<0$ for every $\eta \geq \eta_{1}>0$. Since $W$ is a finite dimensional subspace, we select an $R=R(W)>0$ such that $\varphi(u) \leq 0$ for any $u \in W$ with $\|u\|_{X} \geq R$. That is, (ii) of Theorem 2.13 holds.

From Theorem 2.13, we know $\varphi$ has infinitely many critical points, that is, the problem (1.1) has infinitely many solutions.

By the similar methods as Theorem 3.3, we also have the following corollary.

Corollary 3.4. Let (A1) and the following condition holds:

(A4) There exists a constant $\beta \in(2, \mu]$ such that

$$
0<\beta F(t, u) \leq u f(t, u)
$$

for every $t \in[0, T]$ and $u \in \mathbb{R} \backslash\{0\}$, where $F(t, u)=\int_{0}^{u} f(t, s) d s$. Moreover, $f(t, u)$ and $I_{i j}(u), i=1,2, j=1,2, \ldots, m$, are odd in $u$.

Then, the problem (1.1) has infinitely many solutions.

\section{EXAMPLES}

In this part, we will give corresponding examples to illustrate the main results in our paper.

Example 4.1. Let $T=B=1, A=-1, t_{1} \in[0,1]$, then consider the following differential equation:

$$
\left\{\begin{array}{l}
u^{(i v)}(t)-u^{\prime \prime}(t)+u(t)=-\sin (u(t)) e^{-\cos (u(t))}-u^{\frac{1}{3}}(t), \quad t \neq t_{1}, \text { a.e. } t \in[0,1], \\
\Delta\left(u^{\prime \prime}\left(t_{1}\right)\right)=-\frac{1}{2} u^{3}\left(t_{1}\right), \\
-\Delta\left(u^{\prime \prime \prime}\left(t_{1}\right)\right)=-\frac{1}{3} u^{3}\left(t_{1}\right), \\
u(0)=u(1)=u^{\prime \prime}\left(0^{+}\right)=u^{\prime \prime}\left(1^{-}\right)=0 .
\end{array}\right.
$$

It is obvious to see $f(t, u)=-\sin (u(t)) e^{-\cos (u(t))}-u^{\frac{1}{3}}(t), I_{11}(u)=-\frac{1}{2} u^{3}$, and $I_{21}(u)=$ $-\frac{1}{3} u^{3}$. Let $\mu=4, \xi_{11}=\frac{1}{8}$, and $\xi_{21}=\frac{1}{12}$, that is (A1) holds. Since $|f(t, u)| \leq e+|u|^{\frac{1}{3}}$, then $F(t, u)=-e^{-\cos (u(t))}-\frac{3}{4} u^{\frac{4}{3}} \leq 0$, that is (A2) and (A3) hold. By Theorem 3.1, the problem (4.1) has at least two solutions.

Example 4.2. Let $T=B=1, A=-1, t_{1} \in[0,1]$, then consider the following differential equation:

$$
\left\{\begin{array}{l}
u^{(i v)}(t)-u^{\prime \prime}(t)+u(t)=-e^{t} u^{\frac{1}{5}}(t), \quad t \neq t_{1}, \text { a.e. } t \in[0,1] \\
\Delta\left(u^{\prime \prime}\left(t_{1}\right)\right)=-e u^{7}\left(t_{1}\right) \\
-\Delta\left(u^{\prime \prime \prime}\left(t_{1}\right)\right)=-e^{2} u^{7}\left(t_{1}\right) \\
u(0)=u(1)=u^{\prime \prime}\left(0^{+}\right)=u^{\prime \prime}\left(1^{-}\right)=0
\end{array}\right.
$$


It is obvious to see $f(t, u)=-e^{t} u^{\frac{1}{5}}(t), I_{11}(u)=-e u^{7}$, and $I_{21}(u)=-e^{2} u^{7}$. Let $\mu=8, \xi_{11}=\frac{e}{8}$, and $\xi_{21}=\frac{e^{2}}{8}$, that is (A1) holds. Since $|f(t, u)| \leq e|u|^{\frac{1}{5}}$, then $F(t, u)=-\frac{5 e^{t}}{6} u^{\frac{6}{5}}(t) \leq 0$, that is, (A2) and (A3) hold. Also, $f(t, u), I_{11}(u)$ and $I_{21}(u)$ are odd in $u$. Thus, by Theorem 3.3, the problem (4.2) has infinitely many solutions.

\section{ACKNOWLEDGMENTS}

This work has been supported by the NSFC (11571088, 12071105), the Zhejiang Provincial Natural Science Foundation of China (LY14A010024), and Scientific Research Fund of Hunan Provincial Education Department (14A098).

\section{REFERENCES}

[1] G.A. Afrouzi, A. Hadjian and V.D. Rădulescu, Variational approach to fourth-order impulsive differential equations with two control parameters, Results Math., 65: 371-384, 2014.

[2] G. Bonanno and B. Di Bella, A boundary value problem for fourth-order elastic beam equations, J. Math. Anal. Appl., 343: 1166-1176, 2008.

[3] A. Cabada and S. Tersian, Existence and multiplicity of solutions to boundary value problems for fourth-order impulsive differential equations, Bound. Value Probl., No. 1, 2014.

[4] D. Gao and J. Li, Three solutions for fourth-order impulsive differential inclusions via nonsmooth critical point theory, J. Funct. Space, 2018: 1-9, 2018.

[5] J. Mawhin and M. Willem, Critical Point Theory and Hamiltonian Systems, Appl. Math. Sci., Springer, Berlin, 1989.

[6] P.H. Rabinowitz, Minimax Methods in Critical Point Theory with Applications to Differential Equations, CBMS Regional Conference Series in Mathematics, American Mathematical Society, Providence, RI, 1986.

[7] J. Sun, H. Chen and L. Yang, Variational methods to fourth-order impulsive differential equations, J. Appl. Math. Comput., 35: 323-340, 2011.

[8] Y. Tian and X. Liu, Applications of variational methods to Sturm-Liouville boundary-value problem for fourth-order impulsive differential equations, Math. Meth. Appl. Sci., 37: 95-105, 2013.

[9] J. Xie and Z. Luo, Solutions to a boundary value problem of a fourth-order impulsive differential equation, Bound. Value Probl., No. 154, 2013.

[10] Y. Yue, Y. Tian, M. Zhang and J. Liu, Existence of infinitely many solutions for fourth-order impulsive differential equations, Appl. Math. Lett., 81: 72-78, 2018.

[11] E. Zeidler, Nonlinear Functional Analysis and its Applications, Springer, Berlin, 1985. 